\title{
Structure-based screening of Natural product libraries in search of potential antiviral drug-leads as first-line treatment to Covid-19 infection
}

Aditya Rao ( $\sim$ adityaraosj@gmail.com )

Central Food Technological Research Institute CSIR https://orcid.org/0000-0002-2546-5109

Nandini Shetty

CSIR-CFTRI: Central Food Technological Research Institute CSIR

\section{Research Article}

Keywords: Drug design, Synthetic drugs, Structural diversity, SARS-CoV-2, Medicinal chemistry

Posted Date: June 28th, 2021

DOI: https://doi.org/10.21203/rs.3.rs-654687/v1

License: () (7) This work is licensed under a Creative Commons Attribution 4.0 International License. Read Full License

Version of Record: A version of this preprint was published at Microbial Pathogenesis on March 1st, 2022. See the published version at https://doi.org/10.1016/j.micpath.2022.105497. 


\section{Abstract}

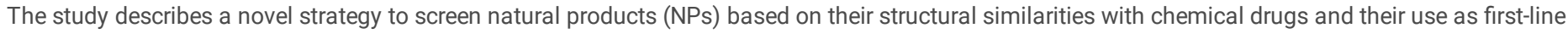

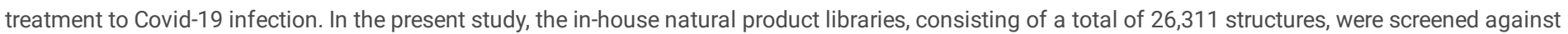

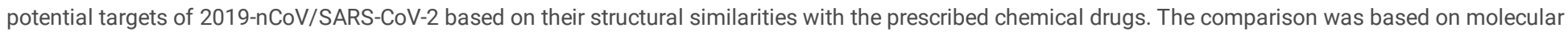

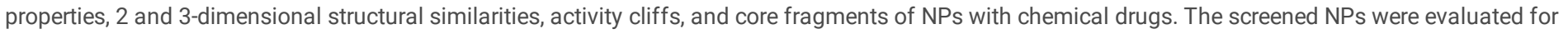

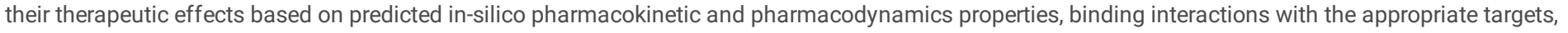

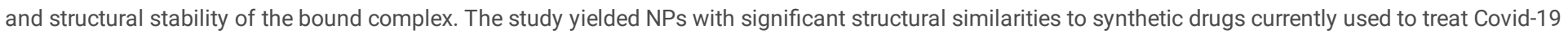

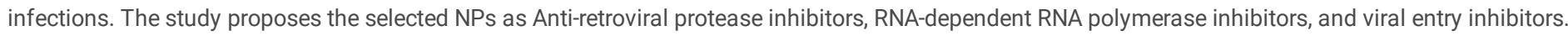

\section{Introduction}

Viral infections play an important role in human diseases, and their regular outbreaks repeatedly underlined the need for their prevention in safeguarding public health [1]. The recent outbreak of the novel coronavirus Covid-19 was declared 'public health emergency of international concern' by World Health Organization (WHO) in view of its severity [2]. The Coronavirus disease (COVID-19), previously known as '2019 novel coronavirus' or '2019-nCoV', is an infectious disease caused by a newly discovered coronavirus; severe acute respiratory syndrome coronavirus 2 or SARS-CoV-2 [3]. The SARS-CoV-2 is a member of the Coronavirinae family belonging to the Betacorona genus [4]. Structurally it is spherical or pleomorphic in shape, with a diameter of about 60$140 \mathrm{~nm}$. All ages are susceptible to COVID-19 infection, and its clinical manifestations range from asymptomatic to mild to severe and even to death depending on the underlying health conditions of individuals $[5,6]$. The most commonly reported symptoms are fever, chills, headache, body aches, dry cough, fatigue, pneumonia, and complicated dyspnea. The virus transmits from person to person via the nasal, oral, eye, and mucosal secretions of the infected patient and direct transmission through the inhalation of droplets released during the patient's cough or sneeze $[7,8]$.

For the clinical diagnosis of SARS-CoV-2, the reverse transcription-quantitative polymerase chain reaction (RT-qPCR) method is widely being used today [9]. It is a nucleic acid detection test where nasopharyngeal and oropharyngeal samples were used for the detection. However, to provide quick diagnosis techniques like transcription loop-mediated isothermal amplification (RT-LAMP), transcription-mediated amplification (TMA), CRISPR-based assays, rolling circle amplification, and microarray hybridization assays have been developed and are currently in use [10,11].

To prevent the transmission of SARS-CoV-2, the development of an effective vaccine is highly essential. Therefore, scientists around the world are engaged in developing potential vaccines. However, at this stage, it is unclear which vaccine strategy would be most effective. Figure-1 describes some of the most widely used vaccines currently developed against Covid-19. The other potential treatment strategies include inhibition of RNA-Dependent RNA Polymerase activity, viral protease inhibition, viral entry inhibition, immune modulation, monoclonal antibodies, janus kinase inhibitors, nutritional supplements, and the conventional plasma therapy (Table 1) [11]. The developmental status of different antiviral drugs to treat Covid-19 conditions is shown in figure 3.

Table 1: Mechanism of action of some of the COVID-19 prescribed drugs and their common usage. 


\begin{tabular}{|c|c|}
\hline COVID-19 prescribed Drugs & Known mechanism of action and their common usage \\
\hline \multicolumn{2}{|c|}{ Inhibiting the RNA-Dependent RNA Polymerase } \\
\hline Remdesivir & Inhibits viral RNA production and replication of EBOV [12] \\
\hline Favipiravir & Anti-influenza drug [13] \\
\hline Galidesivir & Hepatitis C treatment [14] \\
\hline Ribavirin & Hepatitis C treatment viral hemorrhagic fevers [15] \\
\hline Sofosbuvir & Hepatitis C treatment [16] \\
\hline \multicolumn{2}{|l|}{ Viral Protease Inhibitors } \\
\hline Lopinavir/Ritonavir & Anti-retroviral protease inhibitor [17] \\
\hline Nelfinavir & Inhibits HIV-1 and HIV-2 retroviral proteases [18] \\
\hline Atazanavir & Anti-retroviral protease inhibitor used to treat HIV infections [19] \\
\hline Darunavir & anti-retroviral protease inhibitor [20] \\
\hline Danoprevir & HCV Protease Inhibitor [21] \\
\hline \multicolumn{2}{|l|}{ Viral Entry Inhibitor } \\
\hline Hydroxychloroquine & Antimalarial drug [22] \\
\hline Arbidol & Anti- influenza drug [23] \\
\hline Ivermectin & Antiviral/antiparasitic drug [24] \\
\hline \multicolumn{2}{|l|}{ Immune Modulators } \\
\hline Interferon-alpha (IFN-2b) & Antiviral and/or anti-neoplastic drug [25] \\
\hline Tacrolimus & Inhibits T-lymphocyte signal transduction and IL-2 transcription [26] \\
\hline \multicolumn{2}{|l|}{ Monoclonal Antibodies } \\
\hline Sarilumab & IL-6 receptor blocker [27] \\
\hline Tocilizumab & $\begin{array}{l}\text { Treatment of rheumatoid arthritis and juvenile idiopathic arthritis. Inhibits the IL-6 signaling } \\
\text { pathway[28] }\end{array}$ \\
\hline \multicolumn{2}{|l|}{ Janus Kinase Inhibitors } \\
\hline Fedratinib & Inhibits JAK2 the treatment of rheumatoid arthritis [29] \\
\hline Baricitinib & Reversible inhibitor of both JAK1 and JAK2 in the treatment of rheumatoid arthritis [30] \\
\hline \multicolumn{2}{|l|}{ Nutritional Supplements } \\
\hline Vitamin C & Boosts immunity by stimulating IFN production [31] \\
\hline Vitamin D & Involved in adaptive immunity, immune cell differentiation, proliferation, and maturation [32] \\
\hline Folic Acid & Important for rapid cell proliferation [33] \\
\hline \multicolumn{2}{|l|}{ Miscellaneous } \\
\hline Valsartan & Angiotensin-converting enzyme (ACE) inhibitors and angiotensin receptor blockers [34] \\
\hline Entresto & Angiotensin II receptor neprilysin inhibitor [35] \\
\hline Telbivudine & Antiviral thymidine nucleoside analog against the hepatitis B virus DNA polymerase [36] \\
\hline Azithromycin & Antibiotic drug [37] \\
\hline Colchicine & Prevent gout attacks [38] \\
\hline Methylprednisolone & Anti-inflammatory drug [39] \\
\hline Naproxen & Anti-inflammatory and antiviral drug used against Influenza A virus [40] \\
\hline Tilorone & Treatment of influenza, acute respiratory viral infection, viral hepatitis, and viral encephalitis [41] \\
\hline Cobicistat & Cytochrome P450 (CYP3A) inhibition [42] \\
\hline Omeprazole & $\begin{array}{l}\text { Proton pump inhibitor used to treat gastroesophageal reflux disease, } \\
\text { heartburn, and ulcers [43] }\end{array}$ \\
\hline Pirfenidone & Antifibrotic and anti-inflammatory drug [44] \\
\hline
\end{tabular}


Natural products and traditional medicines have been serving as the greatest source for modern drug discovery. Their derivatives are recognized for many years as the source of the therapeutic potential and structural diversity. There are over 200,000 compounds reported in the scientific literature. NPs are more often structurally complex, with well-organized structure and steric properties offering efficacy, efficiency, and selectivity of molecular targets [50]. However, their utilization on many health conditions is well documented; it is in the hands of existing traditional practitioners and herbologists to define their applications for newly emerging diseases. The biological activities reported from different plant extracts often narrow down to pre-reported molecules rather than novel compounds [51], creating a real challenge to medicinal chemists. In this avenue, the search for new therapeutic molecules is the need of the hour to combat against new health challenges.

The biological activity of any molecule is attributed to its structural arrangements. If two molecules have a similar structure, they will most probably have a similar biological effect [52-54] (Fig.3). The computational chemists are successful in exploiting this principle for the construction of diverse compound libraries and select compounds for high-throughput screening experiments [52]. Computational advancements with the introduction of parallel processing clusters, cloud-based computing, and highly effective graphical processing units (GPUs), tremendous success has been achieved in the field of modern drug discovery [55]. The knowledge of natural products and ligands, earlier used as starting points for drug discovery, has greatly influenced computational biology techniques [56]. These advancements have been speeded up by the creation of new algorithms for more accurate predictions, simulations, and interpretations [57-62]. The extensive molecular dynamics (MD) simulations can provide insights into the host-virus interactions, disease spread, and possible regulative/preventive mechanisms [63]. The present study proceeds to identify natural products as first-line treatment options for Covid-19 infections in this avenue. By considering the structural properties of prescribed chemical drugs currently used to treat different Covid-19 conditions, natural product libraries were screened to identify potential antiviral drug molecules. The study extends to describe the possible mechanism of their therapeutic actions creating new opportunities for nature-based therapeutics.

\section{Materials And Methods}

\section{Dataset collection and library construction:}

An in-house natural product library consisting of 26,311 natural product structures was constructed using natural products information from different databases like Dr. Duke's database (https://phytochem.nal.usda.gov/phytochem/search) [64], Phytochemical Interactions Database (http://www.genome.jp/db/pcidb), and Natural product activity and species source database (NPASS) (http://bidd.group/NPASS/index.php) [65]. The natural product library was further categorized as flavans (339), flavones (193), and isoflavonoids (457), and the rest of the molecules as a general group. The broadspectrum antiviral drugs currently under investigation to treat Covid-19 conditions were collected from the drugvirus.info server (https://drugvirus.info). For comparison, the small molecule synthetic drugs were categorized into molecules present in Pubchem Covid19 portal

(306) [66] (https://pubchem.ncbi.nlm.nih.gov/\#query=covid-19), and molecules present at different stages of clinical trials (138) (As of 31 ${ }^{\text {st }}$ August 2020) based on the available information from ClinicalTrials.gov database [67] (https://www.clinicaltrials.gov/ct2/home). Further, the study was extended to compare the most promising investigational drugs like Remdesivir, Arbidol, Lopinavir, and Ritonavir. The top 10 structures most similar to investigational drugs were selected for in-silico PK/PD analysis and HTVS studies.

\section{Structure-based screening of Natural products:}

The non-redundant natural product libraries were compared against chemical drugs currently under prescription/study to treat COVID 19 infection. The comparison is based on 2 and 3-dimensional structural similarities, activity cliffs (ACs), and core fragments (CFs). The structural similarities were assessed based on the number of fragments that both molecules have to the number of fragments found in any two structures [68]. The structural scaffolds (SSs) were analyzed based on plane ring system to determine the sub-structures. ACs, CFs, and SSs were determined employing Osiris DataWarrior V.4.4.3 software [68].

\section{Molecular properties based PK/PD analysis:}

Natural products are the major source of oral drugs 'beyond Lipinski's rule of five' [69-71]. The druglikeness assessment, pharmacokinetic (PK), and pharmacodynamics (PD) of NPs were determined based on their molecular properties like molecular weight, cLogP, hydrogen atom donors, hydrogen atom acceptors, and rotatable hydrogen bonds. These properties are used as filtering parameters to estimate the oral bioavailability, solubility, and permeability of new drug candidates $[69,71,72]$. The natural products obtained from structural comparison were considered as hits for in-silico PK/PD assessment. Molecular properties were predicted using Osiris Data warrior V.4.4.3 software [68]. The admetSAR server [73] was used to predict different parameters constituting the PK/PD properties of the selected molecules.

\section{Molecular interactions studies using automate docking:}


Automated docking was performed to deduce the binding interactions of selected natural products with appropriate target proteins. Broyden-Fletcher-GoldfarbShanno algorithm implemented in the AutoDockVina was employed to study proper binding modes of the selected natural products in different

conformations [74]. The antiviral drugs currently being prescribed for Covid-19 first-line treatment were retrieved from the drugvirus.info server, and their action mechanisms were studied using the Inxight: Drugs database (https://drugs.ncats.io/) (Table 1).

Based on the action mechanism of the standard drugs, HIV-1 protease I50V isolate, influenza virus hemagglutinin, SARS-CoV NSP12 polymerase and HIV-1 protease A02 isolate were selected for the docking studies. The protein structures were retrieved from protein databank (https://www.rcsb.org/) and were prepared for docking studies. For each target, residues forming the binding site were identified using the PDBsum server. The antiviral drug; Lopinavir and its related natural products were docked against anti-retroviral protease inhibitor (I50V isolate) (PDB ID 30XV), Ritonavir and its related natural products were docked against anti-retroviral protease inhibitor (A02 isolate) (PDB ID 4NJV), Remdesivir, and its related natural products were docked against anti-retroviral protease inhibitor (PDB ID 7BV2), and Arbidol and its related natural products were docked against anti-retroviral protease inhibitor (PDB ID 5T6S). For the ligand molecules, all the torsions were allowed to rotate during docking. The in-silico studies were performed on a local machine equipped with AMD Ryzen 5 six-core 3.4 GHz processor, 8GB graphics, and 16 GB RAM with Microsoft Windows 10 and Ubuntu 16.04 LTS dual boot operating systems.

\section{Molecular dynamic simulations to predict the protein structural stability:}

the structural stability of the free and bound targets was assessed using MD simulations run for a time scale of $20 \mathrm{~ns}[75,76]$ by employing the GROMOS96 54a7 [77] force field implemented in the GROMACS-2018 package [78]. A periodic cubic solvated box was created around the target proteins with at least $10 \AA$ distance from the edge of the box and solvated using the simple point charge (SPC) model [79] and neutralized using sodium and chloride ions. Temperature coupling at $300 \mathrm{~K}$ was done using V-rescale thermostat [80], and pressure coupling at $10^{5} \mathrm{~Pa}$ was done using Parrinello-Rahman barostat [81]. Bond parameters were adjusted using the LINCS algorithm [82], and the particle mesh Ewald method (PME) [83] was used to evaluate electrostatic interactions. The final MD trajectories were prepared for a time scale of $20 \mathrm{~ns}$ at a time step of $2 \mathrm{fs}$ with trajectory coordinates updated at $10 \mathrm{ps}$ intervals. The final trajectories were analyzed using gmx energy, gmx rms, gmx rmsf, gmx gyrate, gmx do_dssp, and gmx sasa modules of GROMACS along with interaction energies in terms of electrostatic and van der Waals energy between the ligand and the macromolecule.

\section{Biding free energy calculations using g_mmpbsa:}

For Molecular mechanics/Poisson-Boltzmann surface area (MMPBSA) calculations, trajectory files were created from the final $10 \mathrm{~ns}$ with coordinates updated every 200ps. The g_mmpbsa package was used for binding energy calculations[84]. The g_mmpbsa package uses the following equation to calculate the binding energy of the protein-ligand complex;

$\Delta \mathbf{G}_{\text {Binding }}=\mathbf{G}_{\text {Complex }}-\left(\mathbf{G}_{\text {Protein }}+\mathbf{G}_{\text {Ligand }}\right)$

The ' $G$ ' term can be further decomposed into the following components-

$\Delta \mathrm{G}=\Delta \mathrm{E}_{\mathrm{MM}}+\Delta \mathrm{G}_{\text {Solvation }}-\mathrm{T} \Delta \mathrm{S}=\Delta \mathrm{E}_{(\text {Bonded }+ \text { Non-bonded })}+\Delta \mathrm{G}_{(\text {Polar }+ \text { Non-polar })}-\mathrm{T} \Delta \mathrm{S}$

Where,

$\mathrm{G}_{\text {Complex }}=$ total free energy of the binding complex,

$\mathrm{G}_{\text {Protein }}$ and $\mathrm{G}_{\text {Ligand }}=$ total free energies of protein and ligand, respectively.

$E_{M M}=$ vacuum potential energy; $G_{\text {Solvation }}=$ free energy of solvation

\section{Results}

\section{Structure-based screening of Natural products:}

The natural product library consisting of 26,311 structures was screened against local Pubchem Covid19 library of Covid 19 prescribed drugs and Covid 19 clinical trials drug library. Among the total number of molecules screened, 17,798 natural product structures were found to have more than $60 \%$ structural similarities against Pubchem Covid19 library, of which 41 molecules were flavans, 41 were flavones, and 272 were isoflavonoids. The comparison against clinical trials drug library yielded 14,689 natural products with more than $60 \%$ structure similarity consisting of 30 flavans, 18 flavones, and 78 isoflavonoids. The study was extended to compare the complete natural product library against the most promising investigational drugs, viz. Remdesivir, Arbidol, Lopinavir, and Ritonavir molecules yielded 35 natural product structures with considerable structural similarity (Table 2).

Table 2: Natural products structurally similar to prescribed Covid-19 drugs and their similarity score. 


\begin{tabular}{|c|c|}
\hline Synthetic drug and the identified NPs & Similarity score \\
\hline \multicolumn{2}{|l|}{ Remdesivir } \\
\hline 12_28_0xa_8_Hydroxy_Manzamin_A & 0.7676 \\
\hline Marineosin_A & 0.7558 \\
\hline Bis(Gorgiacerol)Amine & 0.8107 \\
\hline Methylstemofoline & 0.7645 \\
\hline Chetracin_B & 0.7877 \\
\hline Oxyprotostemonine & 0.7644 \\
\hline Stemocurtisine & 0.7569 \\
\hline Munroniamide & 0.7705 \\
\hline Alstolobine_A & 0.7598 \\
\hline Discorhabdin_H & 0.7665 \\
\hline \multicolumn{2}{|l|}{ Arbidol } \\
\hline Phellibaumin_A & 0.6838 \\
\hline Difloxacin & 0.7363 \\
\hline Lamellarin_D & 0.7175 \\
\hline Lamellarin_Gamma_Acetate & 0.6682 \\
\hline Hydroxy-6-Methylpyran-2-One_Derivative & 0.6822 \\
\hline Cyathuscavin_C & 0.7363 \\
\hline Cyathusal_B & 0.7135 \\
\hline Clausarin & 0.6770 \\
\hline Cyathuscavin_B & 0.7135 \\
\hline Pulvinatal & 0.7010 \\
\hline \multicolumn{2}{|l|}{ Lopinavir } \\
\hline Hexahydrodipyrrol derivative & 0.7458 \\
\hline Beauvericin & 0.7478 \\
\hline Chaetocin & 0.7615 \\
\hline Mollenine_A & 0.7462 \\
\hline Chetracin_B & 0.7877 \\
\hline Beauvericin_H1 & 0.7549 \\
\hline Dragonamide_A & 0.7703 \\
\hline Chetracin_D & 0.7892 \\
\hline Dimethyl-3-Oxodecanamide derivative & 0.7569 \\
\hline Symplocamide_A & 0.7481 \\
\hline \multicolumn{2}{|l|}{ Ritonavir } \\
\hline Bionectin_B & 0.7146 \\
\hline Luteoalbusin_A & 0.6906 \\
\hline Bionectin_A & 0.7122 \\
\hline Oidioperazine_A & 0.7153 \\
\hline Holstiine & 0.6876 \\
\hline Chetracin_B & 0.7142 \\
\hline Mollenine_A & 0.6796 \\
\hline Methaniminium derivative & 0.7274 \\
\hline Verticillin_E & 0.7181 \\
\hline
\end{tabular}

Page 6/23 


\section{Molecular properties based PK/PD analysis:}

Molecular properties and Pharmacokinetics prediction of natural products were predicted using Osiris data warrior software and the admetSAR server. The druglikeness estimated based on the molecular properties of the selected structures indicated that out of 35 molecules, 23 molecules with positive scores indicated their potential drug-like effects. Gastrointestinal (GI) absorption is an important parameter to screen orally administered drugs. A positive value shown in Table 3A for gastrointestinal (GI) absorption suggests a high probability of success for absorption into the intestinal tract [85]. While the blood-brain barrier (BBB) penetration indicates the potentials of a drug to cross into the brain, it can bind to specific receptors and activate specific signaling pathways. Therefore, the prediction of BBB penetration is crucial in the drug development pipeline [86]. In the present study, 33 molecules were found to penetrate the human intestine barrier, 17 molecules penetrating the blood-brain barrier, and none of them being the substrate for Cytochromes P450 group of isozymes which regulates drug metabolism, indicating a high possibility of their bioavailability (Table $3 \mathrm{~A}$ ). Further, out of 35 molecules, 34 were predicted to be nonmutagenic and non-tumorigenic and non-irritant, with 10 molecules predicted to have reproductive effects (Table 3B). Among the 35 structures, 29 compounds were non-AMES toxic, 34 non-carcinogens, and 34 were not readily biodegradable.

Table 3: A) Molecular properties and Pharmacokinetics prediction of natural products filtered in for screening against COVID-19 condition. 


\begin{tabular}{|c|c|c|c|c|c|c|c|c|c|c|}
\hline \multirow[t]{2}{*}{ Identified NPs } & \multicolumn{8}{|c|}{ Bioavailability and Druglikeness } & \multicolumn{2}{|c|}{ In silico Pharmac } \\
\hline & cLogP & Mol. wt & $\begin{array}{l}\mathrm{H}- \\
\text { Acceptors }\end{array}$ & $\begin{array}{l}\mathrm{H}- \\
\text { Donors }\end{array}$ & $\begin{array}{l}\text { Rotatable } \\
\text { Bonds }\end{array}$ & $\begin{array}{l}\text { Total } \\
\text { Surface } \\
\text { Area }\end{array}$ & $\begin{array}{l}\text { Polar } \\
\text { Surface } \\
\text { Area }\end{array}$ & Druglikeness & $\begin{array}{l}\text { Human } \\
\text { intestinal } \\
\text { absorption }\end{array}$ & $\begin{array}{l}\text { Ca } \\
\text { pel }\end{array}$ \\
\hline 12_28_0xa_8_Hydroxy_Manzamin_A & 5.2547 & 562.755 & 6 & 2 & 1 & 414.34 & 60.33 & -2.227 & $0.696+$ & 0.5 \\
\hline Alstolobine_A & 2.4707 & 398.457 & 7 & 1 & 6 & 297.41 & 80.86 & -8.1671 & $0.988+$ & 0.5 \\
\hline Beauvericin & 5.2239 & 783.96 & 12 & 0 & 9 & 610.8 & 139.83 & 4.3764 & $0.991+$ & 0.6 \\
\hline Beauvericin_H1 & 5.3247 & 801.95 & 12 & 0 & 9 & 617.15 & 139.83 & 3.0364 & $0.990+$ & 0.5 \\
\hline Bionectin_A & 2.9631 & 450.542 & 7 & 3 & 1 & 282.66 & 139.27 & 5.5488 & $0.889+$ & 0.5 \\
\hline Bionectin_B & 2.6488 & 494.595 & 8 & 4 & 2 & 311.68 & 159.5 & 5.0182 & $0.900-$ & 0.5 \\
\hline Bis(Gorgiacerol)Amine & 5.4399 & 757.83 & 13 & 3 & 10 & 560.06 & 183.97 & -19.005 & $0.965-$ & 0.6 \\
\hline Chaetocin & 2.7962 & 696.852 & 12 & 4 & 3 & 409.82 & 246.96 & 5.8356 & $0.900+$ & 0.6 \\
\hline Chetracin_B & 1.092 & 760.916 & 14 & 6 & 3 & 437.49 & 312.72 & 5.4873 & $0.885+$ & 0.5 \\
\hline Chetracin_D & 0.3976 & 788.99 & 14 & 6 & 7 & 496.04 & 287.42 & 5.6124 & $0.922+$ & 0.5 \\
\hline Clausarin & 5.9768 & 380.482 & 4 & 1 & 4 & 295.44 & 55.76 & -5.9217 & $0.975+$ & 0.8 \\
\hline Cyathusal_B & 0.5256 & 346.29 & 8 & 3 & 3 & 243.75 & 122.52 & -4.326 & $0.915+$ & 0.5 \\
\hline Cyathuscavin_B & 0.5053 & 376.316 & 9 & 3 & 4 & 264.22 & 131.75 & -4.7328 & $0.878+$ & 0.6 \\
\hline Cyathuscavin_C & 0.0774 & 362.289 & 9 & 4 & 3 & 248.31 & 142.75 & -2.2479 & $0.868+$ & 0.5 \\
\hline Difloxacin & 1.251 & 399.396 & 6 & 1 & 3 & 283.48 & 64.09 & 5.1997 & $0.985+$ & 0.8 \\
\hline Discorhabdin_H & -10.123 & 762.664 & 10 & 3 & 5 & 337.61 & 198.19 & 2.7192 & $0.734+$ & 0.6 \\
\hline Dragonamide_A & 3.7111 & 653.905 & 10 & 2 & 18 & 539.59 & 125.32 & -3.0172 & $0.969+$ & 0.5 \\
\hline Hexahydrodipyrrol derivative & 0.2123 & 427.456 & 9 & 3 & 2 & 288.36 & 119.41 & 6.7335 & $0.946+$ & 0.6 \\
\hline Holstiine & 1.5964 & 382.458 & 6 & 1 & 0 & 270.15 & 70.08 & 5.6428 & $0.972+$ & 0.6 \\
\hline $\begin{array}{l}\text { Hydroxy-6-Methylpyran-2- } \\
\text { One_Derivative }\end{array}$ & 5.228 & 500.586 & 8 & 4 & 11 & 387.58 & 141.36 & -13.889 & $0.984+$ & 0.5 \\
\hline Lamellarin_D & 4.3105 & 499.474 & 9 & 3 & 4 & 352.71 & 119.09 & 1.8379 & $0.983+$ & 0.6 \\
\hline Lamellarin_Gamma_Acetate & 5.3 & 573.596 & 10 & 1 & 8 & 423.68 & 106.84 & 2.3739 & $0.987+$ & 0.6 \\
\hline Luteoalbusin_A & 3.1416 & 464.569 & 7 & 3 & 2 & 295.59 & 139.27 & 5.9847 & $0.890+$ & 0.5 \\
\hline Marineosin_A & 4.6896 & 409.572 & 5 & 2 & 2 & 323.4 & 62.4 & -2.232 & $0.986+$ & 0.6 \\
\hline Methaniminium derivative & -0.4649 & 910.463 & 21 & 10 & 14 & 679.31 & 329.1 & 6.1103 & $0.795+$ & 0.6 \\
\hline Methylstemofoline & 0.9975 & 345.394 & 6 & 0 & 1 & 220.03 & 57.23 & 4.0559 & $0.922+$ & 0.6 \\
\hline Mollenine_A & 3.3511 & 368.475 & 5 & 1 & 4 & 275.46 & 58.64 & 3.6919 & $0.980+$ & 0.5 \\
\hline Munroniamide & -0.4894 & 597.663 & 12 & 2 & 7 & 419.88 & 166.86 & -8.9989 & $0.940+$ & 0.6 \\
\hline Oidioperazine_A & 1.9865 & 538.647 & 9 & 3 & 4 & 357.08 & 167.78 & 6.2082 & $0.843+$ & 0.5 \\
\hline Oxyprotostemonine & 1.004 & 431.483 & 8 & 0 & 2 & 289.89 & 83.53 & 2.3627 & $0.890+$ & 0.6 \\
\hline Phellibaumin_A & 2.8483 & 352.297 & 7 & 4 & 2 & 248.17 & 120.36 & 0.0022 & $0.952+$ & 0.8 \\
\hline Pulvinatal & 0.9535 & 360.317 & 8 & 2 & 4 & 259.66 & 111.52 & -6.9354 & $0.919+$ & 0.6 \\
\hline Stemocurtisine & 1.4247 & 347.41 & 6 & 0 & 1 & 239.64 & 57.23 & 2.9196 & $0.922+$ & 0.6 \\
\hline Symplocamide_A & 0.6976 & 1052.03 & 23 & 11 & 18 & 763.41 & 359.52 & 1.3524 & $0.915+$ & 0.6 \\
\hline Verticillin_E & 1.7482 & 752.872 & 14 & 4 & 3 & 439.8 & 281.1 & 4.5639 & $0.895+$ & 0.5 \\
\hline
\end{tabular}

Table 3: B) In-silico Pharmacodynamics prediction of natural products selected for screening against COVID-19 condition. 


\begin{tabular}{|c|c|c|c|c|c|c|c|}
\hline Identified NPs & Mutagenic & Tumorigenic & $\begin{array}{l}\text { Reproductive } \\
\text { effective }\end{array}$ & $\begin{array}{l}\text { Ocular } \\
\text { irritancy }\end{array}$ & $\begin{array}{l}\text { Aerobic } \\
\text { biodegradibility }\end{array}$ & $\begin{array}{l}\text { Ames } \\
\text { tooxicity score }\end{array}$ & Carcinogen \\
\hline 12_28_0xa_8_Hydroxy_Manzamin_A & NONE & NONE & NONE & $0.946-$ & $1.00-$ & $0.707-$ & $0.607-$ \\
\hline Alstolobine_A & NONE & $\mathrm{HIGH}$ & NONE & $0.979-$ & $1.00-$ & $0.714-$ & $0.573-$ \\
\hline Beauvericin & NONE & NONE & NONE & $0.925-$ & $0.912-$ & $0.772-$ & $0.622-$ \\
\hline Beauvericin_H1 & NONE & NONE & NONE & $0.922-$ & $0.996-$ & $0.776-$ & $0.536-$ \\
\hline Bionectin_A & NONE & NONE & NONE & $0.972-$ & $0.986-$ & $0.733-$ & $0.609-$ \\
\hline Bionectin_B & NONE & NONE & NONE & $0.965-$ & $0.988-$ & $0.870-$ & $0.611-$ \\
\hline Bis(Gorgiacerol)Amine & NONE & NONE & $\mathrm{HIGH}$ & $0.901-$ & $0.623-$ & $0.573-$ & $0.487-$ \\
\hline Chaetocin & NONE & NONE & NONE & $0.918-$ & $0.994-$ & $0.645-$ & $0.623-$ \\
\hline Chetracin_B & NONE & NONE & NONE & $0.911-$ & $0.973-$ & $0.679-$ & $0.644-$ \\
\hline Chetracin_D & NONE & NONE & NONE & $0.904-$ & 0.996- & $0.678-$ & $0.627-$ \\
\hline Clausarin & NONE & NONE & $\mathrm{HIGH}$ & $0.607+$ & 0.993- & $0.506-$ & $0.472-$ \\
\hline Cyathusal_B & NONE & NONE & $\mathrm{HIGH}$ & $0.561-$ & $0.937-$ & $0.707+$ & $0.465-$ \\
\hline Cyathuscavin_B & NONE & NONE & $\mathrm{HIGH}$ & $0.590-$ & $0.966-$ & $0.712+$ & $0.515+$ \\
\hline Cyathuscavin_C & NONE & NONE & $\mathrm{HIGH}$ & $0.574-$ & 0.937- & $0.707+$ & $0.465-$ \\
\hline Difloxacin & NONE & NONE & NONE & $0.949-$ & $1.00-$ & $0.885+$ & $0.610-$ \\
\hline Discorhabdin_H & NONE & NONE & NONE & $0.960-$ & $1.00-$ & $0.593-$ & $0.532-$ \\
\hline Dragonamide_A & NONE & NONE & NONE & $0.922-$ & $1.00-$ & $0.812-$ & $0.678-$ \\
\hline Hexahydrodipyrrol derivative & NONE & NONE & NONE & $0.927-$ & $1.00-$ & $0.658-$ & $0.597-$ \\
\hline Holstiine & NONE & NONE & NONE & $0.986-$ & $0.951-$ & $0.572-$ & $0.501-$ \\
\hline $\begin{array}{l}\text { Hydroxy-6-Methylpyran-2- } \\
\text { One_Derivative }\end{array}$ & NONE & NONE & NONE & $0.732-$ & $0.500+$ & $0.815-$ & $0.723-$ \\
\hline Lamellarin_D & NONE & NONE & $\mathrm{HIGH}$ & $0.833-$ & $0.993-$ & $0.586-$ & $0.389-$ \\
\hline Lamellarin_Gamma_Acetate & NONE & NONE & $\mathrm{HIGH}$ & $0.989-$ & $0.995-$ & $0.880-$ & $0.599-$ \\
\hline Luteoalbusin_A & NONE & NONE & NONE & $0.986-$ & $0.987-$ & $0.670-$ & $0.630-$ \\
\hline Marineosin_A & NONE & NONE & NONE & $0.972-$ & $1.00-$ & $0.655-$ & $0.651-$ \\
\hline Methaniminium derivative & NONE & NONE & NONE & $0.905-$ & $0.962-$ & $0.615-$ & $0.570-$ \\
\hline Methylstemofoline & NONE & NONE & NONE & $0.891-$ & $1.00-$ & $0.755-$ & $0.470-$ \\
\hline Mollenine_A & NONE & NONE & NONE & $0.986-$ & 0.997- & $0.572-$ & $0.528-$ \\
\hline Munroniamide & LOW & $\mathrm{HIGH}$ & LOW & $0.978-$ & $1.00-$ & $0.512-$ & $0.562-$ \\
\hline Oidioperazine_A & NONE & NONE & NONE & $0.987-$ & 0.997- & $0.670-$ & $0.606-$ \\
\hline Oxyprotostemonine & NONE & NONE & NONE & $0.943-$ & $0.994-$ & $0.681-$ & $0.440-$ \\
\hline Phellibaumin_A & $\mathrm{HIGH}$ & NONE & $\mathrm{HIGH}$ & $0.528-$ & $0.911-$ & $0.550+$ & $0.419-$ \\
\hline Pulvinatal & NONE & NONE & $\mathrm{HIGH}$ & $0.547-$ & $0.966-$ & $0.712+$ & $0.515+$ \\
\hline Stemocurtisine & NONE & NONE & NONE & $0.914-$ & $0.995-$ & $0.781-$ & $0.420-$ \\
\hline Symplocamide_A & NONE & NONE & NONE & $0.901-$ & $0.945-$ & $0.644-$ & $0.594-$ \\
\hline Verticillin_E & NONE & NONE & $\mathrm{HIGH}$ & $0.900-$ & $0.986-$ & $0.763-$ & $0.610-$ \\
\hline
\end{tabular}

\section{Molecular interactions studies using automate docking:}

The in-silico molecular interaction studies were used to predict the most effective natural product drug to bind to the appropriate target involved in the regulation of virus entry, replication, assembly and release, as well as host-specific interactions. In the present study, the docking studies were carried for synthetic antiviral agents as well as their structurally similar natural products against different targets proteins of SARS-CoV-2 to deduce the structural insight of molecular interactions. The study yielded natural products being effectively bound to their respective targets (Table 4). The results were expressed in terms of docking energy $(\mathrm{kcal} / \mathrm{mol})$. Many of the selected natural products have displayed docking energies higher than their structurally similar standard drug counterparts. The natural products structurally similar to Remdesivir interact with SARS-CoV NSP12 polymerase with docking energies comparably higher than the standard drug. The natural products tested as influenza virus hemagglutinin inhibitors are also bound to the target with docking energies higher than 
the standard drug arbodol. The binding interactions of natural products tested as viral protease inhibitors were compared with standard drugs lopinavir and ritonavir. Further, their molecular interactions were found stabilized by the formation of many hydrogen bonds. The effectiveness of these binding of natural product with highest interaction energy in each group was selected for protein stability assessment using molecular dynamics simulations (Fig. 4).

Table 4: Molecular interactions between the selected natural products with targets of their structurally similar chemical drugs expressed as docking energies along with their structure similarity score. 


\begin{tabular}{|c|c|c|c|c|}
\hline Target protein & Synthetic drug and the identified NPs & $\begin{array}{l}\text { Docking } \\
\text { Energy* }\end{array}$ & $\begin{array}{l}\mathrm{H}- \\
\text { bonds }\end{array}$ & Interacting Residues \\
\hline \multirow[t]{11}{*}{ SARS-CoV NSP12 POLYMERASE } & Remdesivir & -7.2 & 03 & ILE23, LEU126, GLY48 \\
\hline & 12_28_0xa_8_Hydroxy_Manzamin_A & -10.4 & 02 & GLY130, ALA38 \\
\hline & Marineosin_A & -7.9 & 00 & - \\
\hline & Bis(Gorgiacerol)Amine & -7.8 & 02 & ILE23, GLY130 \\
\hline & Methylstemofoline & -7.7 & 02 & SER128, ALA129 \\
\hline & Chetracin_B & -7.5 & 01 & PHE156 \\
\hline & Oxyprotostemonine & -7.5 & 02 & SER128, ALA129 \\
\hline & Stemocurtisine & -7.5 & 01 & GLY48 \\
\hline & Munroniamide & -6.9 & 05 & VAL49, ILE131, GLY48, GLY130, LEU126 \\
\hline & Alstolobine_A & -6.8 & 03 & PHE156, ASP157, ALA154 \\
\hline & Discorhabdin_H & -6.7 & 02 & GLY48, ASP22 \\
\hline \multirow{11}{*}{$\begin{array}{l}\text { INFLUENZA VIRUS } \\
\text { HEMAGGLUTININ }\end{array}$} & Arbidol & -7.1 & 01 & GLU64 \\
\hline & Phellibaumin_A & -9.4 & 04 & ASP280, SER290, LYS58, ILE288 \\
\hline & Difloxacin & -8.4 & 03 & LYS58, LEU292, PRO293 \\
\hline & Lamellarin_D & -8.4 & 02 & LYS58, CYS305 \\
\hline & Lamellarin_Gamma_Acetate & -7.8 & 01 & GLU57 \\
\hline & $\begin{array}{l}\text { Hydroxy-6-Methylpyran-2- } \\
\text { One_Derivative }\end{array}$ & -7.6 & 03 & THR59, GLU57, THR59 \\
\hline & Cyathuscavin_C & -7.5 & 02 & GLU57, PR0306 \\
\hline & Cyathusal_B & -7.4 & 02 & GLU57, PR0306 \\
\hline & Clausarin & -7.3 & 02 & GLU64, ARG85 \\
\hline & Cyathuscavin_B & -7.3 & 00 & - \\
\hline & Pulvinatal & -7.3 & 01 & THR59 \\
\hline \multirow[t]{11}{*}{ HIV-1 PROTEASE I50V ISOLATE } & Lopinavir & -6.5 & 03 & GLY49, GLY51, GLY52 \\
\hline & Hexahydrodipyrrol derivative & -8.4 & 03 & PR081, ASP25, GLY48 \\
\hline & Beauvericin & -7.2 & 01 & GLY49 \\
\hline & Chaetocin & -7.1 & 06 & $\begin{array}{l}\text { THR74, ASN88, GLN92, ASP30, ILE72, } \\
\text { GLY73 }\end{array}$ \\
\hline & Mollenine_A & -7.1 & 00 & - \\
\hline & Chetracin_B & -6.9 & 00 & - \\
\hline & Beauvericin_H1 & -6.6 & & VAL50, GLY51, THR80 \\
\hline & Dragonamide_A & -6.3 & 02 & ASP30, VAL50 \\
\hline & Chetracin_D & -6.2 & 04 & THR74, ARG87, ASP29, GLY73 \\
\hline & Dimethyl-3-0xodecanamide derivative & -5.6 & 03 & VAL50, GLY51, PHE53 \\
\hline & Symplocamide_A & -4.6 & 00 & - \\
\hline \multirow[t]{8}{*}{ HIV-1 PROTEASE A02 ISOLATE } & Ritonavir & -7.7 & 04 & ASP29, ASP30, GLY48, GLY49 \\
\hline & Bionectin_B & -8.1 & 03 & ILE50, THR82, GLY51 \\
\hline & Luteoalbusin_A & -8.0 & 04 & GLY51, GLY52, PR081, PR079 \\
\hline & Bionectin_A & -7.7 & 02 & THR96, ASN98 \\
\hline & Oidioperazine_A & -7.7 & 02 & ILE50, ASP25 \\
\hline & Holstiine & -7.1 & 00 & - \\
\hline & Chetracin_B & -7.0 & 02 & ARG87, LUE97 \\
\hline & Mollenine_A & -6.9 & 00 & - \\
\hline
\end{tabular}

Page $11 / 23$ 


\begin{tabular}{|l|lll|l} 
Methaniminium derivative & -6.6 & 01 & PR081 \\
\hline Verticillin_E & -6.6 & 02 & THR74, ASN88 \\
\hline Chaetocin & -6.4 & 02 & ARG08, THR26 \\
\hline
\end{tabular}

${ }^{*} \mathrm{kcal} / \mathrm{mol}$

Molecular dynamic simulations to predict the protein structural stability:

In the present study, united-atom MD simulations were performed to confirm the accuracy of binding resulted from docking studies. The result of the MD simulation displayed the conformational changes acquired by different target proteins of SARS-CoV-2 upon binding and inferred the structural insight on molecular stability (fig 4).

The RMSD analysis was done to understand the deviation of $\mathrm{Ca}$ atoms of the protein from its backbone, and RMSF analysis was done to study the fluctuations associated with the amino acid residues of the protein during the simulation. The average RMS deviations and RMS fluctuations were calculated from the MD trajectories of natural product, and synthetic drug bound HIV-1 protease (I50V isolate), Influenza virus haemagglutinin, SARS-CoV NSP 12 polymerase, and HIV-1 protease (A02 isolate) and were compared with their respective unbound structures. Lesser RMS deviations were observed in the bound structure of HIV-1 protease (I50V isolate) after the binding of Hexahydropyrrolo Derivative compared to Lopinavir standard drug. The protein SARS-CoV NSP 12 polymerase displayed lesser RMS deviations after the binding of HydroxyManzamin_A. In comparison, HIV-1 protease (A02 isolate) exhibited lesser RMS deviations after the binding of Bionectin_B compared to their respective chemical drug counterparts. RMS deviations were lower in Arbidol bound Influenza virus haemagglutinin than natural product Phellibaurin_A bound structure (Fig. 4a-d). Lesser RMS fluctuations were observed in the natural product bound structures of HIV-1 protease, Influenza virus haemagglutinin, and HIV-1 protease than their respective chemical drug bound structures (fig.4e-h). From the RMDF plots, it can be inferred that, though the residues displayed higher fluctuations at certain positions, the protein was able to retain its secondary structure's packability. This was inferred based on the Rg plots (Fig.4i-I), where the structures were found to be very tightly packed, as the secondary structure elements like $\alpha$-helix, $\beta$-sheet, and turn, were remodelled at each time step of the MD simulation. The SASA plots (Fig. 4m-p) also supported these findings.

The binding free energy calculations performed using the g_mmpba module displayed better binding of natural products with their respective target proteins compared to their chemical drug counterparts. The binding free energies of 12_28_Oxa_8_Hydroxy_Manzamin_A (-56.19kJ/mol), Phellibaurin_A

$(-125.49 \mathrm{~kJ} / \mathrm{mol})$, and Hexahydropyrrolo Derivative $(-91.66 \mathrm{~kJ} / \mathrm{mol})$ were found to be higher than their respective structurally similar standard drug counterparts; remdesivir (-48.74kJ/mol), arbidol (-102.17), and lopinavir $(-81.19 \mathrm{~kJ} / \mathrm{mol})$ indicating their firm binding with their respective targets. However, the standard drug ritonavir displayed a higher binding energy of $-180.82 \mathrm{~kJ} / \mathrm{mol}$ compared to its structurally similar natural product bionectin $\mathrm{B}(-162.08 \mathrm{~kJ} / \mathrm{mol}$. The associated terms for binding free energy calculations along with the calculated MD parameters for unbound and ligand-bound targets detailing RMSD, RMSF, Rg, SASA, Secondary structure, Coul-SR energy, and LJ-SR energy are detailed in table 5.

Table 5: Calculated MD parameters for native and ligand-bound SARS CoV2 drug targets obtained from the MD simulation along with binding energies and the contributing energy terms of the prescribed drugs and their most similar natural product calculated using g_mmpbsa module. 


\begin{tabular}{|c|c|c|c|c|c|c|c|c|c|c|c|}
\hline \multirow{3}{*}{$\begin{array}{l}\text { Gromacs } \\
\text { Modules }\end{array}$} & & \multicolumn{3}{|c|}{ SARS-CoV NSP12 POLYMERASE } & \multicolumn{3}{|c|}{$\begin{array}{l}\text { INFLUENZA VIRUS } \\
\text { HEMAGGLUTININ }\end{array}$} & \multicolumn{3}{|c|}{ HIV-1 PROTEASE I50V ISOLATE } & \multirow{3}{*}{$\begin{array}{l}\mathrm{HI} \\
\mathrm{N} \\
\mathrm{Pr}\end{array}$} \\
\hline & & Native & Remdesivir & $\begin{array}{l}\text { Hydroxy } \\
\text { Manzamin A }\end{array}$ & Native & Arbidol & Phellibaurin_A & Native & Lopinavir & Hexahydropyrrolo & \\
\hline & & \multicolumn{3}{|l|}{ Protein } & \multicolumn{3}{|l|}{ Protein } & \multicolumn{3}{|l|}{ Protein } & \\
\hline & $\begin{array}{l}\text { Potential } \\
\text { Energy }\end{array}$ & -0.638 & -0.638 & -0.637 & -4.605 & -4.604 & -4.604 & -0.436 & -0.434 & -0.436 & -0 . \\
\hline & $\left(\times 10^{-6}\right)$ & & & & & & & & & & \\
\hline & RMSD (nm) & 0.213 & 0.195 & 0.186 & 0.481 & 0.429 & 0.549 & 0.247 & 0.270 & 0.254 & $0 .:$ \\
\hline & RMSF (nm) & 0.105 & 0.055 & 0.099 & 0.176 & 0.216 & 0.231 & 0.130 & 0.141 & 0.130 & $0:$ \\
\hline & $\mathrm{Rg}(\mathrm{nm})$ & 1.558 & 1.523 & 1.524 & 2.802 & 2.835 & 2.763 & 1.316 & 1.307 & 1.342 & $1 .:$ \\
\hline & SASA $\left(\mathrm{nm}^{2}\right)$ & 92.95 & 85.00 & 87.13 & 175.24 & 176.47 & 177.43 & 59.57 & 60.05 & 60.92 & 64 \\
\hline & $\begin{array}{l}\text { Secondary } \\
\text { Structure }\end{array}$ & 210.49 & 221.97 & 219.29 & 283.92 & 295.63 & 285.29 & 119.47 & 112.07 & 118.78 & 11 \\
\hline & Coul-SR* & - & -47.22 & -3.84 & - & -9.45 & -65.80 & - & -40.29 & -30.24 & - \\
\hline & LJ-SR* & - & -92.72 & -64.15 & - & -109.61 & -114.98 & - & -113.62 & -109.54 & - \\
\hline \multirow[t]{5}{*}{$\begin{array}{l}\text { MMPBSA } \\
\text { Module }\end{array}$} & $\begin{array}{l}\text { Binding } \\
\text { Energy* }\end{array}$ & - & -48.74 & -56.19 & - & -102.17 & -125.49 & - & -81.19 & -91.66 & - \\
\hline & $\begin{array}{l}\text { SASA } \\
\text { Energy* }\end{array}$ & - & -18.86 & -8.23 & - & -13.52 & -52.63 & - & -14.04 & -14.10 & - \\
\hline & $\begin{array}{l}\text { Polar } \\
\text { Solvation } \\
\text { Energy* }\end{array}$ & - & 177.89 & 32.97 & - & 43.77 & 129.78 & - & 98.18 & 70.62 & - \\
\hline & $\begin{array}{l}\text { Electrostatic } \\
\text { Energy** }\end{array}$ & - & -68.45 & -4.00 & - & -7.75 & -13.76 & - & -29.35 & -15.37 & - \\
\hline & $\begin{array}{l}\text { van der } \\
\text { Waals } \\
\text { Energy* }\end{array}$ & - & -139.31 & -76.93 & - & -124.66 & -62.11 & - & -135.98 & -132.81 & - \\
\hline
\end{tabular}

* $\mathrm{kJ} / \mathrm{mol}$

\section{Discussion}

Viral infections have always been creating challenges in human healthcare research. The recent outbreak of navel Coronavirus disease, Covid-19, due to the advent of globalization and ease of travel has underscored the need for prevention and safeguarding public health [1]. Despite the advancements in modern drug research, many viruses lack preventive vaccines or effective therapies. In addition, the constant mutations undergone by the virus made it highly [87] challenging for scientists. Further, the potential development of drug-resistant mutants, especially for viral enzyme-specific inhibitors, have significantly hampered the drug efficacy $[1,88,89]$. Therefore, identifying efficacious and cost-effective antiviral drugs in the absence of potential vaccines or standard therapies is of utmost importance. Herbal medicines and purified natural products have been serving as an excellent source for modern drug research programs. The mechanistic elucidation of antiviral drug actions has shed light on the viral life cycle, including their entry, replication, assembly and release, and host-specific interactions.

Due to the advancements in virology, molecular biology, and computational biology, we were quickly able to decipher the patho-physiology of Covid-19 infection [87]. This was followed by pharmacological investigations, drug repurposing and vaccine development. Enormous Covid-19 related publications and treatment strategies shows that scientists are trying every possible possibilities to find cure for this infection [11].

The computational models have been designed to predict the interactions of potential human target proteins with specific viral strains. By relying on the available interaction information, these models predict the novel host-virus interactions. These predictions have been reliable in the past in understanding the infection mechanism of SARS-CoV [90], MERS-CoV [90], Ebola virus [91], and Zika virus [92]. However, these computational methods play a significant role in modern drug research; the experimental verifications of virus-host interactions are needed to substantiate the potential interactions. Along with this, the availability of verified interactions and relevant information is a prerequisite for computational drug discovery methods.

Natural products can be an important complementary medicine to combat against viral infections. Their origin, availability, safety, and cost-effectiveness make them a better choice than synthetic drugs [93]. The present study suggests natural products can exert their therapeutic effects similar to their synthetic drug counterparts. Molecular interaction studies suggests that natural products 12_28_Oxa_8_Hydroxy_Manzamin_A, Marineosin_A, Bis(Gorgiacerol)Amine, Methylstemofoline, Chetracin_B, Oxyprotostemonine, and Stemocurtisine can inhibit RNA-Dependent RNA Polymerase activity similar to remdesivir by binding with SARS-CoV NSP12 polymerase enzyme. Further, all the ten molecules identified to be structurally similar to arbidol displayed binding energies higher than arbidol, suggesting viral entry inhibitory effects. The interactions of natural products structurally similar to Lopinavir and Ritonavir can act as viral protease inhibitors. 
The structural stability imposed by the selected natural products after binding to their respective targets supports their effective binding. Several studies have shown that some natural products can interact with key viral proteins associated with virulence [11,94-97]. Nevertheless, the screening and selection methods that rely on the structural representations involving physiochemical properties, topological indices, molecular graphs, pharmacophore features, molecular shapes, molecular fields, or quantitative measures are expected to reduce false-positive results and yield more effective structures. In this avenue, the current research compares natural products with synthetic drugs and proposes the probable mechanism of action, suggesting a reliable option for first-line treatment against Covid-19 infection.

\section{Abbreviations}

ADME- Absorption, Distribution, Metabolism, and Excretion; APBS- Adaptive Poisson- Boltzmann Solver; HTVS- High Throughput Virtual Screening; MDMolecular Dynamics; MM- Molecular mechanical; MMPBSA- Molecular mechanics/Poisson-Boltzmann surface area; NCATS- National Center for Advancing Translational Sciences; NPASS- Natural product activity and species source database; NPs - Natural products; PD- Pharmacodynamics; PDB- Protein Data Bank; PK

Pharmacokinetics; PME- Particle Mesh Ewald method; Rg- Radius of Gyration; RMSD- Root Mean Square Deviation; RMSF- Root Mean Square Fluctuation; R05- Rule-of-Five; SASA-Solvent Accessible Surface Area; SMILES- Simplified Molecular Input Line Entry System; SPC- Simple Point Charge; TPSATopological polar surface area.

\section{Declarations}

Ethics approval and consent to participate: Not applicable.

Consent for publication: Not applicable.

Availability of data and materials: All the data used during the current study are available from the corresponding author on reasonable request.

Competing interests: The authors declare that they have no conflicts of interest.

Availability of data and materials: All the data used during the current study are available from the corresponding author on reasonable request.

Funding: This work is an extension of a research project supported by the Department of Science and Technology (DST)- Science and Engineering Research Board (SERB), Govt. of India (Grant number: PDF/2018/00237).

Author contribution: ARSJ: designed and conceived the study, performed the research and wrote the manuscript. NPS: participated in the results discussion and technical support. Both the authors read and approved the final manuscript.

Acknowledgment: The authors thank the Department of Science and Technology (DST) - Science and Engineering Research Board (SERB), Govt. of India for their financial support.

\section{References}

1. Lin LT, Hsu WC, Lin CC (2014) Antiviral natural products and herbal medicines. J Tradit Complement Med 4:24-35. https://doi.org/10.4103/22254110.124335

2. Li X, Wang W, Zhao X, et al (2020) Transmission dynamics and evolutionary history of 2019-nCoV. J Med Virol 92:501-511. https://doi.org/10.1002/jmv.25701

3. WHO (2021) Global research on coronavirus disease (COVID-19)

4. Rehman SU, Shafique L, Ihsan A, Liu Q (2020) Evolutionary trajectory for the emergence of novel coronavirus SARS-CoV-2. Pathogens 9:. https://doi.org/10.3390/pathogens 9030240

5. Harcourt J, Tamin A, Lu X, et al (2020) Severe acute respiratory syndrome coronavirus 2 from patient with coronavirus disease, United States. Emerg Infect Dis 26:1266-1273. https://doi.org/10.3201/EID2606.200516

6. Wang W, Tang J, Wei F (2020) Updated understanding of the outbreak of 2019 novel coronavirus (2019-nCoV) in Wuhan, China. J Med Virol 92:441447. https://doi.org/10.1002/jmv.25689

7. Chan JFW, Yuan S, Kok KH, et al (2020) A familial cluster of pneumonia associated with the 2019 novel coronavirus indicating person-to-person transmission: a study of a family cluster. Lancet 395:514-523. https://doi.org/10.1016/S0140-6736(20)30154-9

8. Li H, Liu Z, Ge J (2020) Scientific research progress of COVID-19/SARS-CoV-2 in the first five months. J. Cell. Mol. Med. 24:6558-6570

9. Udugama B, Kadhiresan P, Kozlowski HN, et al (2020) Diagnosing COVID-19: The Disease and Tools for Detection. ACS Nano 14:3822-3835

10. Carter LJ, Garner L V., Smoot JW, et al (2020) Assay Techniques and Test Development for CoVID-19 Diagnosis. ACS Cent Sci 6:591-605. https://doi.org/10.1021/acscentsci.0c00501

Page $14 / 23$ 
11. Chilamakuri R, Agarwal S (2021) COVID-19: Characteristics and Therapeutics. Cells 10

12. Tchesnokov EP, Feng JY, Porter DP, Götte M (2019) Mechanism of inhibition of ebola virus RNA-dependent RNA polymerase by remdesivir. Viruses 11: https://doi.org/10.3390/v11040326

13. Coomes EA, Haghbayan H (2020) Favipiravir, an antiviral for COVID-19? J. Antimicrob. Chemother. 75:2013-2014

14. Evans GB, Tyler PC, Schramm VL (2018) Immucillins in Infectious Diseases. ACS Infect. Dis. 4:107-117

15. Gong S, Su J, Yan X, et al (2020) Antiviral therapy for Coronavirus disease 2019. J. Cent. South Univ. (Medical Sci. 45:598-602

16. Wang XX, Luo BF, Jiang HJ, et al (2018) Recovery of natural killer cells is mainly in post-treatment period in chronic hepatitis $\mathrm{C}$ patients treated with sofosbuvir plus ledipasvir. World J. Gastroenterol. 24:4554-4564

17. Li F, Lu J, Ma X (2012) CPY3A4-mediated lopinavir bioactivation and its inhibition by ritonavir. Drug Metab Dispos 40:18-24. https://doi.org/10.1124/dmd.111.041400

18. Musarrat F, Chouljenko V, Dahal A, et al (2020) The anti-HIV drug nelfinavir mesylate (Viracept) is a potent inhibitor of cell fusion caused by the SARSCoV-2 spike (S) glycoprotein warranting further evaluation as an antiviral against COVID-19 infections. J Med Virol 92:2087-2095. https://doi.org/10.1002/jmv.25985

19. Li G, De Clercq E (2020) Therapeutic options for the 2019 novel coronavirus (2019-nCoV). Nat. Rev. Drug Discov. 19:149-150

20. Chen J, Xia L, Liu L, et al (2020) Antiviral activity and safety of darunavir/Cobicistat for the treatment of COVID-19. Open Forum Infect Dis 7:. https://doi.org/10.1093/ofid/ofaa241

21. Chen H, Zhang Z, Wang L, et al (2020) First Clinical Study Using HCV Protease Inhibitor Danoprevir to Treat Naive and Experienced COVID-19 Patients. medRxiv 2020.03.22.20034041. https://doi.org/10.1101/2020.03.22.20034041

22. Satarker S, Ahuja T, Banerjee M, et al (2020) Hydroxychloroquine in COVID-19: Potential Mechanism of Action Against SARS-CoV-2. Curr. Pharmacol. Reports 6:203-211

23. Vankadari N (2020) Arbidol: A potential antiviral drug for the treatment of SARS-CoV-2 by blocking trimerization of the spike glycoprotein. Int J Antimicrob Agents 56:. https://doi.org/10.1016/j.ijantimicag.2020.105998

24. Bray M, Rayner C, Noël F, et al (2020) Ivermectin and COVID-19: A report in Antiviral Research, widespread interest, an FDA warning, two letters to the editor and the authors' responses. Antiviral Res. 178

25. Zhou Q, Chen V, Shannon CP, et al (2020) Interferon-a2b Treatment for COVID-19. Front Immunol 11:. https://doi.org/10.3389/fimmu.2020.01061

26. Russell B, Moss C, George G, et al (2020) Associations between immune-suppressive and stimulating drugs and novel COVID-19 - A systematic review of current evidence. Ecancermedicalscience 14

27. Tu YF, Chien CS, Yarmishyn AA, et al (2020) A review of sars-cov-2 and the ongoing clinical trials. Int. J. Mol. Sci. 21

28. Masiá M, Fernández-González M, Padilla S, et al (2020) Impact of interleukin-6 blockade with tocilizumab on SARS-CoV-2 viral kinetics and antibody responses in patients with COVID-19: A prospective cohort study. EBioMedicine 60:. https://doi.org/10.1016/j.ebiom.2020.102999

29. Wu D, Yang XO (2020) TH17 responses in cytokine storm of COVID-19: An emerging target of JAK2 inhibitor Fedratinib. J Microbiol Immunol Infect 53:368-370. https://doi.org/10.1016/j.jmii.2020.03.005

30. Zhang X, Zhang Y, Qiao W, et al (2020) Baricitinib, a drug with potential effect to prevent SARS-COV-2 from entering target cells and control cytokine storm induced by COVID-19. Int. Immunopharmacol. 86

31. Baladia E, Pizarro AB, Ortiz-Muñoz L, Rada G (2020) Vitamin C for COVID-19: A living systematic review. Medwave 20:e7978.

https://doi.org/10.5867/medwave.2020.06.7978

32. Mohan M, Cherian JJ, Sharma A (2020) Exploring links between Vitamin D deficiency and covid-19. PLoS Pathog 16:.

https://doi.org/10.1371/journal.ppat.1008874

33. Acosta-Elias J, Espinosa-Tanguma R (2020) The Folate Concentration and/or Folic Acid Metabolites in Plasma as Factor for COVID-19 Infection. Front Pharmacol 11:. https://doi.org/10.3389/fphar.2020.01062

34. Aronson JK, Ferner RE (2020) Drugs and the renin-angiotensin system in covid-19. BMJ 369

35. Menendez JT (2016) The Mechanism of Action of LCZ696. Card Fail Rev 2:40. https://doi.org/10.15420/cfr.2016:1:1 
36. Rismanbaf A (2020) Potential Treatments for COVID-19; a Narrative Literature Review. Arch Acad Emerg Med 8:2-4.

https://doi.org/10.22037/aaem.v8i1.596

37. Echeverría-Esnal D, Martin-Ontiyuelo C, Navarrete-Rouco ME, et al (2021) Azithromycin in the treatment of COVID-19: a review. Expert Rev. Anti. Infect. Ther. 19:147-163

38. Parra-Medina R, Sarmiento-Monroy JC, Rojas-Villarraga A, et al (2020) Colchicine as a possible therapeutic option in COVID-19 infection. Clin. Rheumatol. 39:2485-2486

39. Liu J, Zheng X, Huang Y, et al (2020) Successful use of methylprednisolone for treating severe COVID-19. J Allergy Clin Immunol 146:325-327. https://doi.org/10.1016/j.jaci.2020.05.021

40. Wongrakpanich S, Wongrakpanich A, Melhado K, Rangaswami J (2018) A comprehensive review of non-steroidal anti-inflammatory drug use in the elderly. Aging Dis. 9:143-150

41. Lane TR, Massey C, Comer JE, et al (2019) Repurposing the antimalarial pyronaridine tetraphosphate to protect against Ebola virus infection. PLoS Negl Trop Dis 13:. https://doi.org/10.1371/journal.pntd.0007890

42. Mathias AA, German P, Murray BP, et al (2010) Pharmacokinetics and pharmacodynamics of gs-9350: A novel pharmacokinetic enhancer without AntiHIV activity. Clin Pharmacol Ther 87:322-329. https://doi.org/10.1038/clpt.2009.228

43. Nitulescu GM, Paunescu H, Moschos SA, et al (2020) Comprehensive analysis of drugs to treat SARS-CoV-2 infection: Mechanistic insights into current COVID-19 therapies (Review). Int. J. Mol. Med. 46:467-488

44. Seifirad S (2020) Pirfenidone: A novel hypothetical treatment for COVID-19. Med Hypotheses 144:. https://doi.org/10.1016/j.mehy.2020.110005

45. Lin MH, Moses DC, Hsieh CH, et al (2018) Disulfiram can inhibit MERS and SARS coronavirus papain-like proteases via different modes. Antiviral Res 150:155-163. https://doi.org/10.1016/j.antiviral.2017.12.015

46. Sanchez-Pernaute O, Romero-Bueno FI, Selva-O'Callaghan A (2020) Why Choose Cyclosporin A as First-line Therapy in COVID-19 Pneumonia. Reumatol. Clin.

47. Soliman A, Fathy A, Khashab S, et al (2013) Sirolimus conversion may suppress viral replication in hepatitis C virus-positive renal transplant candidates. Exp Clin Transplant 11:408-411. https://doi.org/10.6002/ect.2013.0017

48. Chen X, Kopecky DJ, Mihalic J, et al (2012) Structure-guided design, synthesis, and evaluation of guanine-derived inhibitors of the elF4E mRNA-cap interaction. J Med Chem 55:3837-3851. https://doi.org/10.1021/jm300037x

49. Yiğenoğlu TN, Hacıbekiroğlu T, Berber İ, et al (2020) Convalescent plasma therapy in patients with COVID-19. J. Clin. Apher. 35:367-373

50. Yuan H, Ma Q, Ye L, Piao G (2016) The traditional medicine and modern medicine from natural products. Molecules 21:

https://doi.org/10.3390/molecules21050559

51. Walsh CT, Tang Y (2017) Natural Product Biosynthesis. The Royal Society of Chemistry

52. Martin YC, Kofron JL, Traphagen LM (2002) Do structurally similar molecules have similar biological activity? J Med Chem 45:4350-4358. https://doi.org/10.1021/jm020155c

53. Gfeller D, Grosdidier A, Wirth M, et al (2014) SwissTargetPrediction: A web server for target prediction of bioactive small molecules. Nucleic Acids Res 42:. https://doi.org/10.1093/nar/gku293

54. Kumar A, Zhang KYJ (2018) Advances in the development of shape similarity methods and their application in drug discovery. Front. Chem. 6

55. Nobile MS, Cazzaniga P, Tangherloni A, Besozzi D (2017) Graphics processing units in bioinformatics, computational biology and systems biology. Brief Bioinform 18:870-885. https://doi.org/10.1093/bib/bbw058

56. Aditya Rao SJ, Ramesh CK, Raghavendra S, Paramesha M (2020) Dehydroabietylamine, A Diterpene from Carthamus tinctorious L. Showing Antibacterial and Anthelmintic Effects with Computational Evidence. Curr Comput Aided Drug Des 16:231-237.

https://doi.org/10.2174/1573409915666190301142811

57. Gange SJ, Golub ET (2016) From smallpox to big data: The next 100 years of epidemiologic methods. Am. J. Epidemiol. 183:423-426

58. Docherty AB, Lone NI (2015) Exploiting big data for critical care research. Curr. Opin. Crit. Care 21:467-472

59. Greene CS, Tan J, Ung M, et al (2014) Big data bioinformatics. J. Cell. Physiol. 229:1896-1900

60. Wasser T, Haynes K, Barron J, Cziraky M (2015) Using "big data" to validate claims made in the pharmaceutical approval process. J Med Econ 18:1013-1019. https://doi.org/10.3111/13696998.2015.1108919

Page 16/23 
61. Raghavendra S, Aditya Rao SJ, Kumar V, Ramesh CK (2015) Multiple ligand simultaneous docking (MLSD): A novel approach to study the effect of inhibitors on substrate binding to PPO. Comput Biol Chem 59:81-86. https://doi.org/10.1016/j.compbiolchem.2015.09.008

62. Janakirama ARS, Shivayogi SM, Satyanarayana JK, Kumaran RC (2020) Characterization of isolated compounds from Morus spp. and their biological activity as anticancer molecules. Biolmpacts. https://doi.org/10.34172/bi.2021.09

63. Arantes PR, Saha A, Palermo G (2020) Fighting covid-19 using molecular dynamics simulations. ACS Cent Sci 6:1654-1656.

https://doi.org/10.1021/acscentsci.0c01236

64. Dr. Duke's phytochemical and ethnobotanical databases. US Dep Agric Agric Res Serv 1992-2016.

https://doi.org/http://dx.doi.org/10.15482/USDA.ADC/1239279

65. Zeng X, Zhang P, He W, et al (2018) NPASS: Natural product activity and species source database for natural product research, discovery and tool development. Nucleic Acids Res 46:D1217-D1222. https://doi.org/10.1093/nar/gkx1026

66. Kim S, Chen J, Cheng T, et al (2019) PubChem 2019 update: Improved access to chemical data. Nucleic Acids Res 47:D1102-D1109.

https://doi.org/10.1093/nar/gky1033

67. U.S. National Institute of Health (NIH), U.S. National Library of Medicine (NLM) (2008) ClinicalTrials.gov database. In: U.S. Natl. Libr. Med. https://clinicaltrials.gov/ct2/home

68. Sander T, Freyss J, Von Korff M, Rufener C (2015) DataWarrior: An open-source program for chemistry aware data visualization and analysis. J Chem Inf Model 55:460-473. https://doi.org/10.1021/ci500588j

69. Lipinski CA, Lombardo F, Dominy BW, Feeney PJ (1997) Experimental and computational approaches to estimate solubility and permeability in drug discovery and development settings. Adv. Drug Deliv. Rev. 23:3-25

70. Doak BC, Over B, Giordanetto F, Kihlberg J (2014) Oral druggable space beyond the rule of 5: Insights from drugs and clinical candidates. Chem. Biol. 21:1115-1142

71. Aditya R, Venugopal T, Jayanna N, et al (2020) Bioactive isolates of Morus species as antibacterial agents and their insilico profiling. Lett Drug Des Discov 17:. https://doi.org/10.2174/1570180817999201104120815

72. Jarrahpour A, Motamedifar M, Zarei M, et al (2010) Petra, Osiris, and Molinspiration Together as a Guide in Drug Design: Predictions and Correlation Structure/Antibacterial Activity Relationships of New N-Sulfonyl Monocyclic $\beta$-Lactams. Phosphorus Sulfur Silicon Relat Elem 185:491-497.

https://doi.org/10.1080/10426500902953953

73. Cheng F, Li W, Zhou Y, et al (2012) AdmetSAR: A comprehensive source and free tool for assessment of chemical ADMET properties. J Chem Inf Model 52:3099-3105. https://doi.org/10.1021/ci300367a

74. Trott O, Olson AJ (2010) Software news and update AutoDock Vina: Improving the speed and accuracy of docking with a new scoring function, efficient optimization, and multithreading. J Comput Chem 31:455-461. https://doi.org/10.1002/jcc.21334

75. Lin J-H (2012) Accommodating Protein Flexibility for Structure-Based Drug Design. Curr Top Med Chem 11:171-178.

https://doi.org/10.2174/156802611794863580

76. Salsbury FR (2010) Molecular dynamics simulations of protein dynamics and their relevance to drug discovery. Curr. Opin. Pharmacol. 10:738-744

77. Schmid N, Eichenberger AP, Choutko A, et al (2011) Definition and testing of the GROMOS force-field versions 54A7 and 54B7. Eur Biophys J 40:843856. https://doi.org/10.1007/s00249-011-0700-9

78. Abraham MJ, Murtola T, Schulz R, et al (2015) Gromacs: High performance molecular simulations through multi-level parallelism from laptops to supercomputers. SoftwareX 1-2:19-25. https://doi.org/10.1016/j.softx.2015.06.001

79. Berendsen HJC, Postma JPM, van Gunsteren WF, Hermans J (1981) Interaction Models for Water in Relation to Protein Hydration. pp 331 -342

80. Bussi G, Donadio D, Parrinello M (2007) Canonical sampling through velocity rescaling. J Chem Phys 126:. https://doi.org/10.1063/1.2408420

81. Parrinello M, Rahman A (1981) Polymorphic transitions in single crystals: A new molecular dynamics method. J Appl Phys 52:7182-7190.

https://doi.org/10.1063/1.328693

82. Hess B, Bekker H, Berendsen HJC, Fraaije JGEM (1997) LINCS: A Linear Constraint Solver for molecular simulations. J Comput Chem 18:1463-1472. https://doi.org/10.1002/(SICI)1096-987X(199709)18:12<1463::AID-JCC4>3.0.CO;2-H

83. Essmann U, Perera L, Berkowitz ML, et al (1995) A smooth particle mesh Ewald method. J Chem Phys 103:8577-8593.

https://doi.org/10.1063/1.470117

Page 17/23 
84. Kumari R, Kumar R, Consortium OSDD, Lynn A (2014) g _ mmpbsa - A GROMACS tool for MM-PBSA and its optimization for high-throughput binding energy calculations. J Chem Inf Model 54:1951-1962

85. Kwofie SK, Broni E, Teye J, et al (2019) Pharmacoinformatics-based identification of potential bioactive compounds against Ebola virus protein VP24. Comput Biol Med 113:. https://doi.org/10.1016/j.compbiomed.2019.103414

86. Suenderhauf C, Hammann F, Huwyler J (2012) Computational prediction of blood-brain barrier permeability using decision tree induction. Molecules 17:10429-10445. https://doi.org/10.3390/molecules170910429

87. Yuki K, Fujiogi M, Koutsogiannaki S (2020) COVID-19 pathophysiology: A review. Clin. Immunol. 215

88. Sheu TG, Deyde VM, Okomo-Adhiambo M, et al (2008) Surveillance for neuraminidase inhibitor resistance among human influenza A and B viruses circulating worldwide from 2004 to 2008. Antimicrob Agents Chemother 52:3284-3292. https://doi.org/10.1128/AAC.00555-08

89. Geretti AM, Armenia D, Ceccherini-Silberstein F (2012) Emerging patterns and implications of HIV-1 integrase inhibitor resistance. Curr. Opin. Infect. Dis. 25:677-686

90. Dyall J, Coleman CM, Hart BJ, et al (2014) Repurposing of clinically developed drugs for treatment of Middle East respiratory syndrome coronavirus infection. Antimicrob Agents Chemother 58:4885-4893. https://doi.org/10.1128/AAC.03036-14

91. Cao H, Zhang Y, Zhao J, et al (2017) Prediction of the Ebola Virus Infection Related Human Genes Using Protein-Protein Interaction Network. Comb Chem High Throughput Screen 20:. https://doi.org/10.2174/1386207320666170310114816

92. Barrows NJ, Campos RK, Powell ST, et al (2016) A Screen of FDA-Approved Drugs for Inhibitors of Zika Virus Infection. Cell Host Microbe 20:259-270. https://doi.org/10.1016/j.chom.2016.07.004

93. Islam MT, Sarkar C, El-Kersh DM, et al (2020) Natural products and their derivatives against coronavirus: A review of the non-clinical and pre-clinical data. Phyther. Res. 34:2471-2492

94. Prasansuklab A, Theerasri A, Rangsinth P, et al (2021) Anti-COVID-19 drug candidates: A review on potential biological activities of natural products in the management of new coronavirus infection. J. Tradit. Complement. Med. 11:144-157

95. Huang J, Tao G, Liu J, et al (2020) Current Prevention of COVID-19: Natural Products and Herbal Medicine. Front. Pharmacol. 11

96. Gasmi A, Chirumbolo S, Peana M, et al (2021) The Role of Diet and Supplementation of Natural Products in COVID-19 Prevention. Biol Trace Elem Res. https://doi.org/10.1007/s12011-021-02623-3

97. Chakravarti R, Singh R, Ghosh A, et al (2021) A review on potential of natural products in the management of COVID-19. RSC Adv. 11:16711-16735

\section{Figures}




\section{Which Covid-19 Vaccines Are Most Widely Used?}

Number of countries using selected Covid-19 vaccines as of February 16, 2021

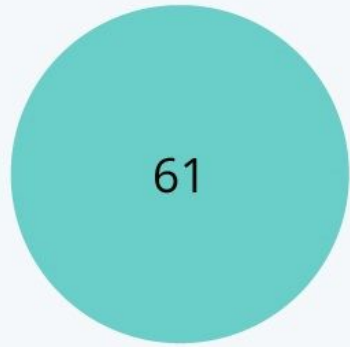

Pfizer

BioNTech

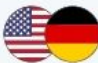

9

Gamaleya (Sputnik)

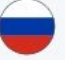

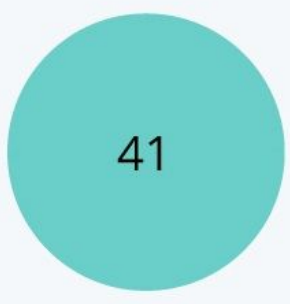

Oxford-

AstraZeneca and

6

Sinovac

$\star 3$

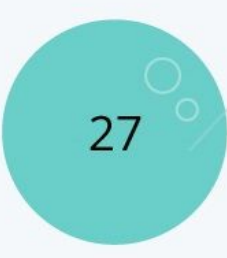

Moderna

플

2

Sinopharm- Bharat Biotech

Wuhan

?.
10

Sinopharm

Beijing

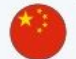

1

(Covaxin)

\section{Figure 1}

Most widely used vaccines currently developed against Covid-19. 


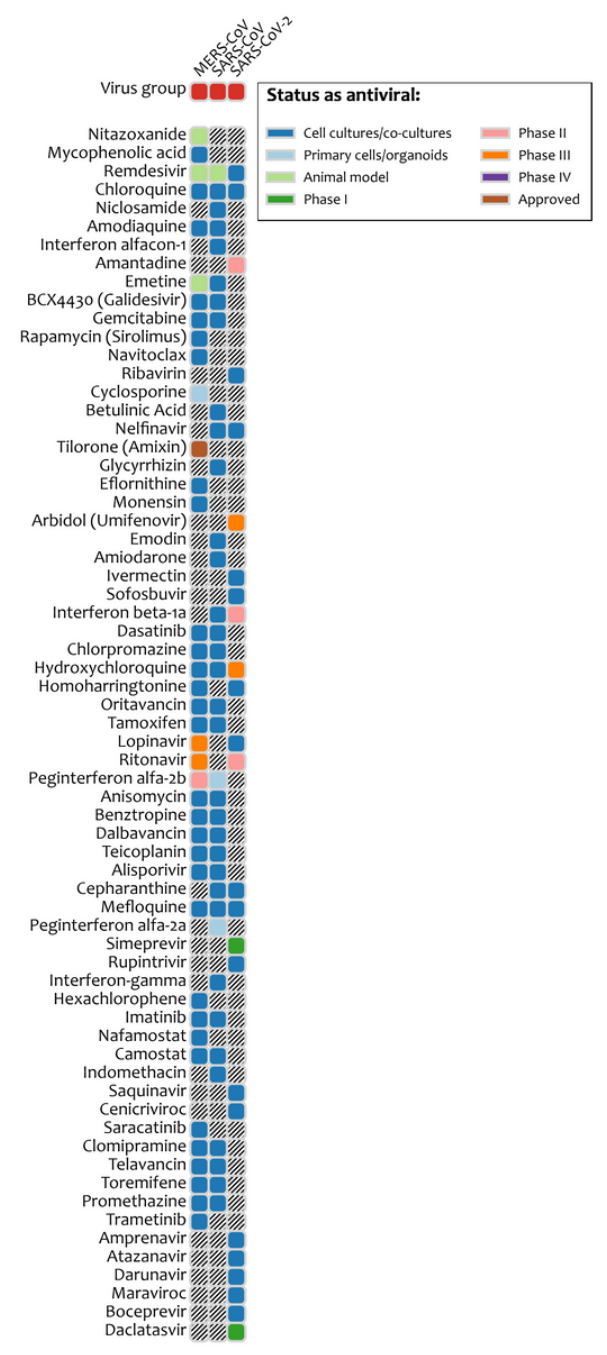

\section{Figure 2}

The broad-spectrum antiviral drugs currently being investigated to treat the Covid-19 condition. 


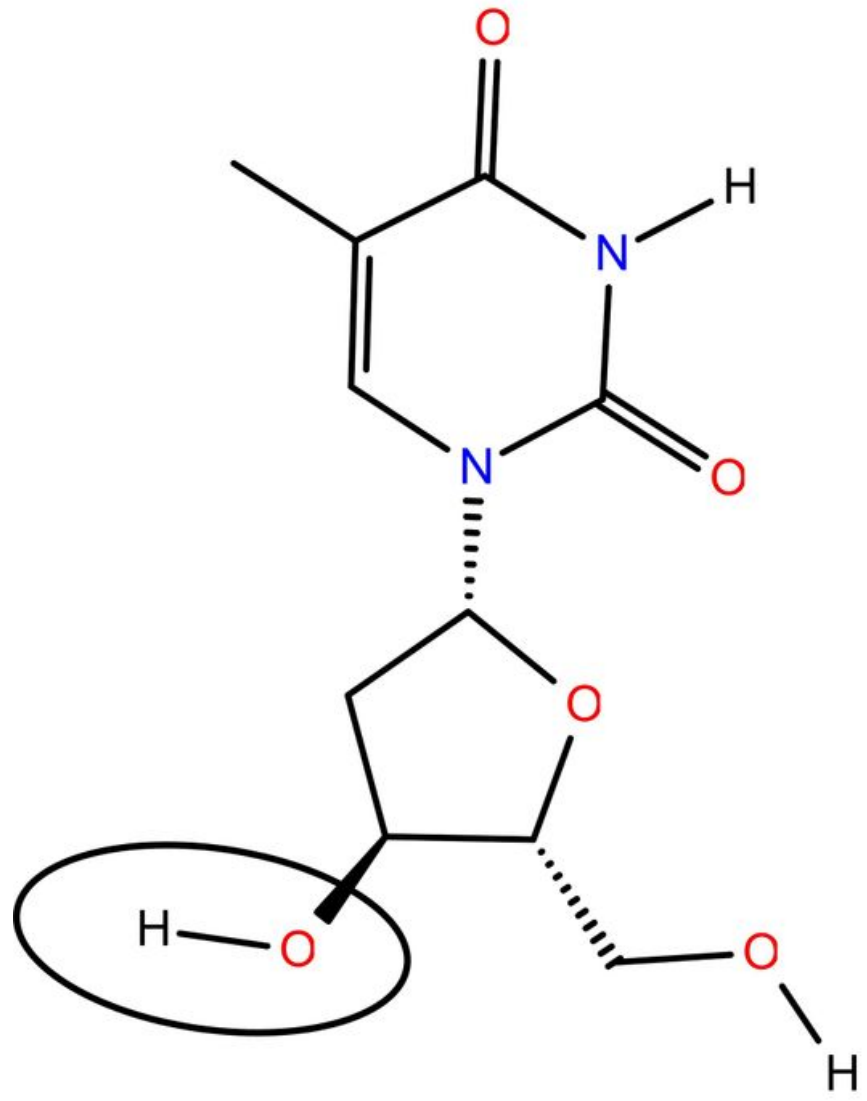

\section{Thymidine}

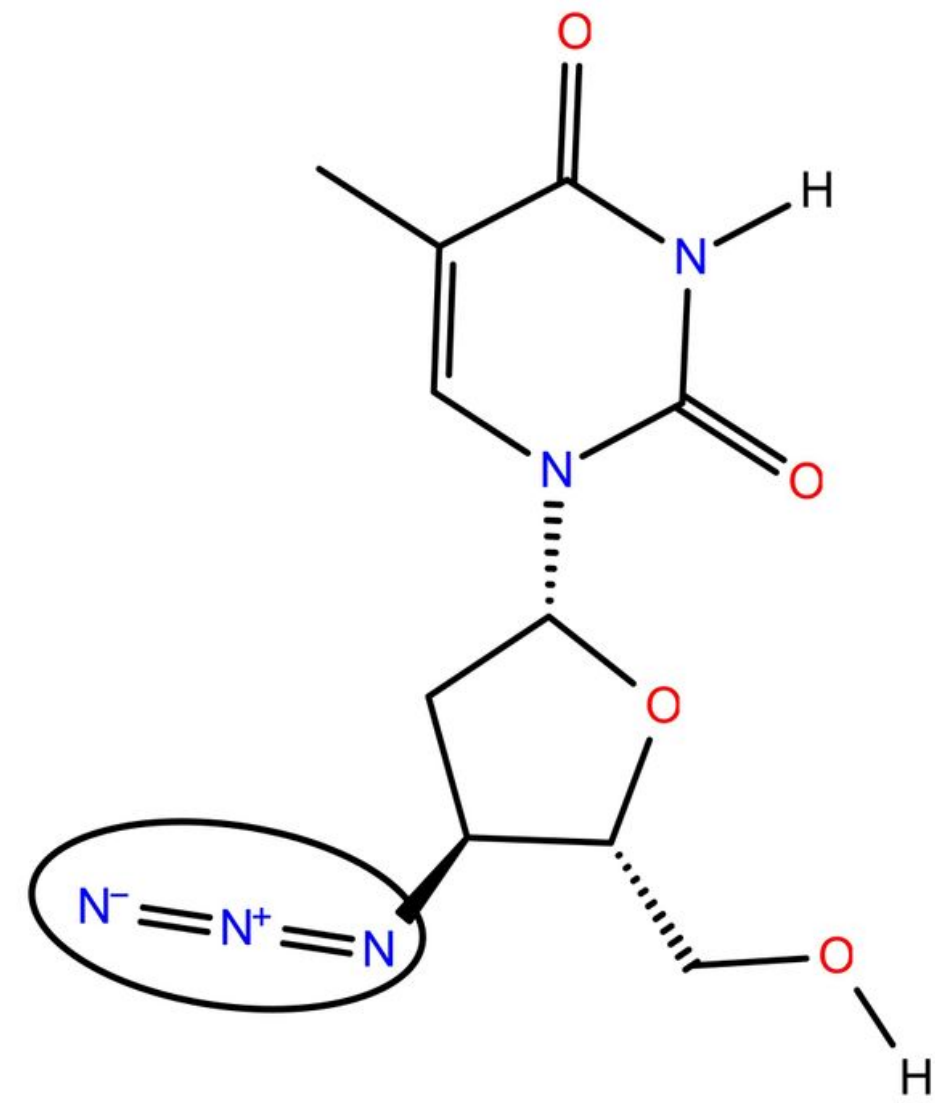

Zidovudine

Figure 3

A comparison describing the structural similarities with variations highlighted inside the ellipse between thymidine, a naturally occurring nucleotide base, and zidovudine, a synthetic drug used to treat HIV patients. Structurally both the molecules share $>93 \%$ identity. 

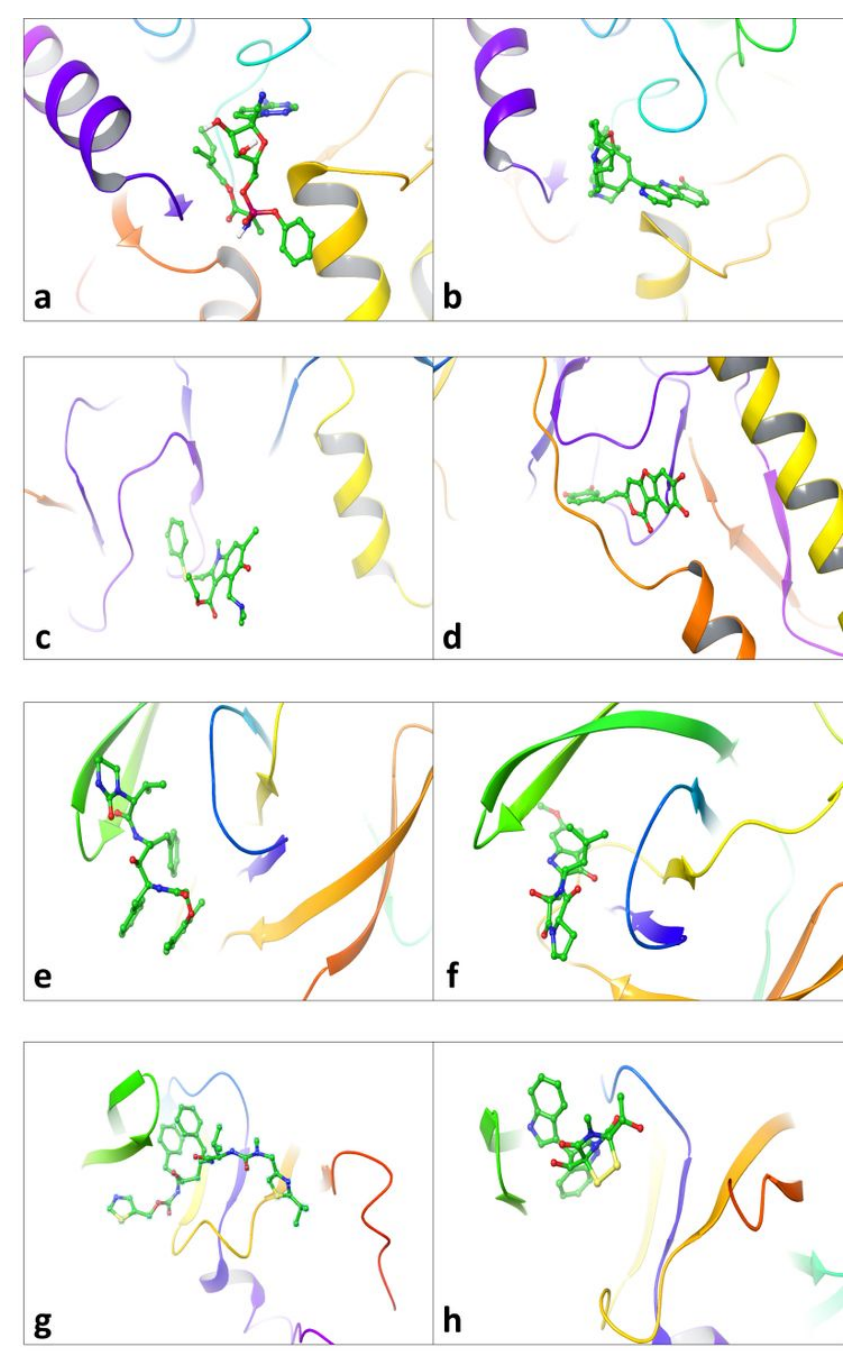

\section{Figure 4}

Docking interaction between Remdesivir (a), and 12_28_0xa_8_Hydroxy_Manzamin_A (b) with SARS-CoV NSP12 polymerase, Arbidol (c), and Phellibaumin_A

(d) with influenza virus hemagglutinin, Lopinavir (e), and Hexahydrodipyrrol derivative (f) with HIV-1 protease I50V isolate and Ritonavir (g), and Bionectin_B

(h) with HIV-1 protease A02 isolate. 

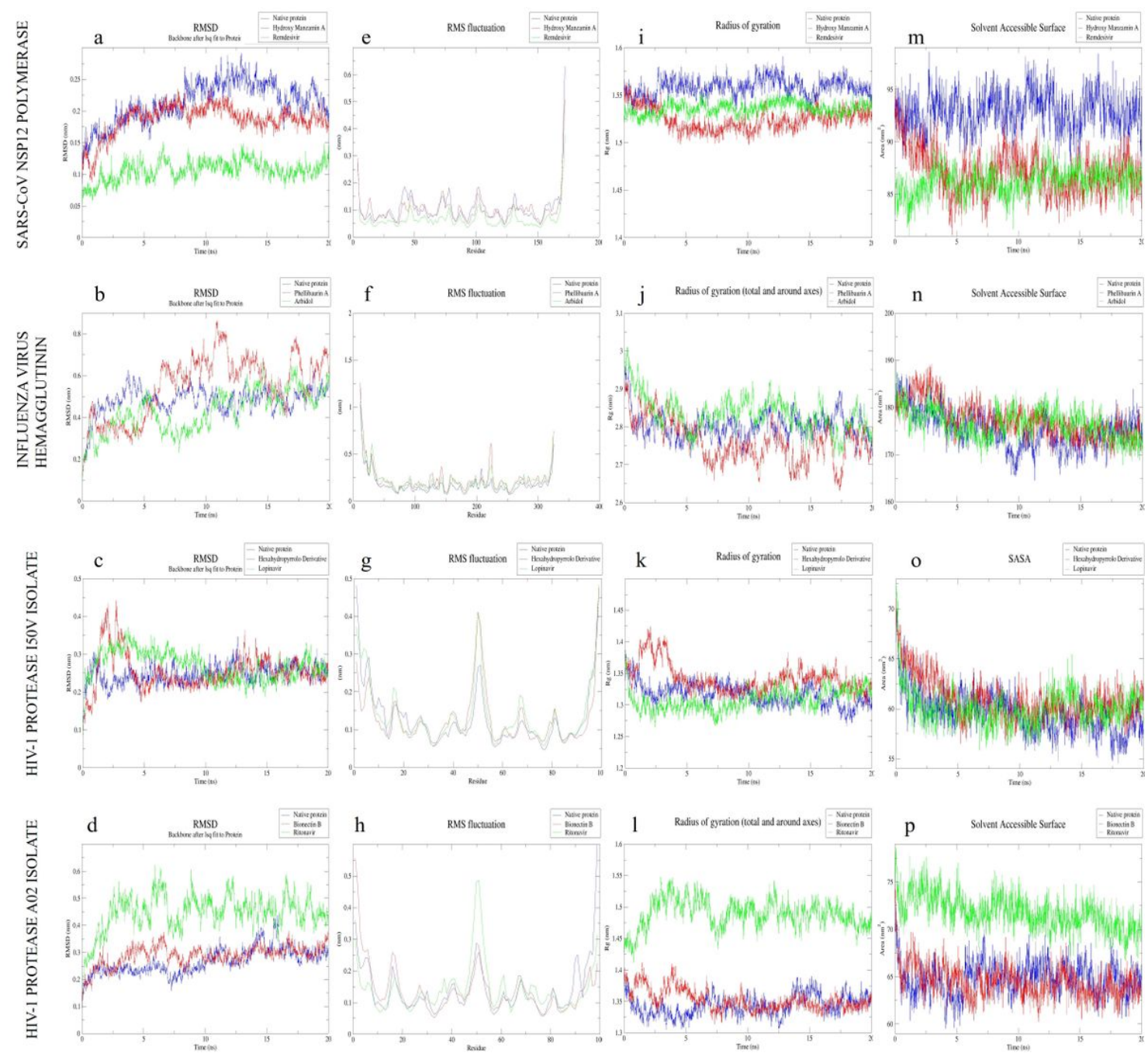

Figure 5

RMSD (a-d), RMSF (e-h), Rg (i-l) and SASA (m-p) plots obtained from MD trajectories analysis of native, Natural product bound, and chemical drug bound structure of SARS-CoV NSP12 polymerase, influenza virus hemagglutinin, , and HIV-1 protease of I50V isolate and A02 isolates. 